\title{
Journal of Gerontology \& Geriatric Research
}

\section{Amyands Hernia: An Unexpected Finding during Inguinal Hernia Repair}

Vinita Angeline Rajadurai*

St John of God Subiaco Hospital, Australia

*Corresponding author: Vinita Angeline Rajadurai, Colorectal Surgical Unit, St John of God Subiaco Hospital, 12 Salvado Road, Subiaco, Western Australia 6008, Australia, Tel: +61452486774; Fax: +61893829888 E-mail: vinita_ar@hotmail.com

Rec date: Jun 17, 2016; Acc date: Jul 27, 2016; Pub date: Jul 30, 2016

Copyright: (c) 2016 Rajadurai VA. This is an open-access article distributed under the terms of the Creative Commons Attribution License, which permits unrestricted use, distribution, and reproduction in any medium, provided the original author and source are credited.

\section{Introduction}

Herniorrhaphy, which is the surgical repair of a hernia, is one of the commonest procedures performed by general surgeons, with over 40,000 hernia operations performed annually in Australia. Approximately 75 percent of abdominal hernias are inguinal and these may contain omentum, bowel and rarely the appendix. The presence of the vermiform appendix, whether inflamed or not, inside the hernia sac is known as Amyand's hernia, which was first described in 1735 by Claudius Amyand, an English surgeon [1]. It is exceedingly rare and occurs in 1 percent of all inguinal hernias with an estimated rate of 0.07-0.13 percent having associated acute appendicitis [2,3]. We report a case whereby an Amyand's hernia was discovered during an elective right-sided inguinal hernia repair.

\section{Case Report}

A 38-year-old Caucasian male was seen at an outpatient surgical clinic with a known right-sided inguinal hernia of more than 2-years, which had recently become symptomatic causing intermittent discomfort with exercise. In addition, following a bout of coughing associated with a viral illness; he noticed protrusion of the hernia extending down into the scrotum that was often difficult to reduce manually. His only other medical history included a vasectomy several years prior.

On examination, his abdomen was soft, non-tender and nondistended. On local examination, a right-sided non-tender irreducible mass with scrotal involvement was present in the right inguinal region.

Elective surgery was planned and an open approach was undertaken. Once the indirect hernia sac was identified, it was dissected away from the spermatic cord to the deep inguinal ring. The indirect hernia sac was then opened and the appendix found lying within, along with adhesions. There were no macroscopic inflammatory changes seen on the appendix as it was dissected off the hernia sac (Figure 1). The appendix measured $121 \mathrm{~mm} \times 13 \mathrm{~mm}$ and a lumen of $2 \mathrm{~mm}$ in diameter. An appendectomy was performed through the inguinal incision only. A right-sided hernioplasty was then performed using the Polypropylene Hernia System mesh.

The patient was discharged home on the following day and returned for routine post-operative follow-up six weeks later with no complications or recurrence. Histopathology of the appendix showed normal mucosa and muscular layers with some evidence of serosal granulation tissue and inflammation (Figure 2).

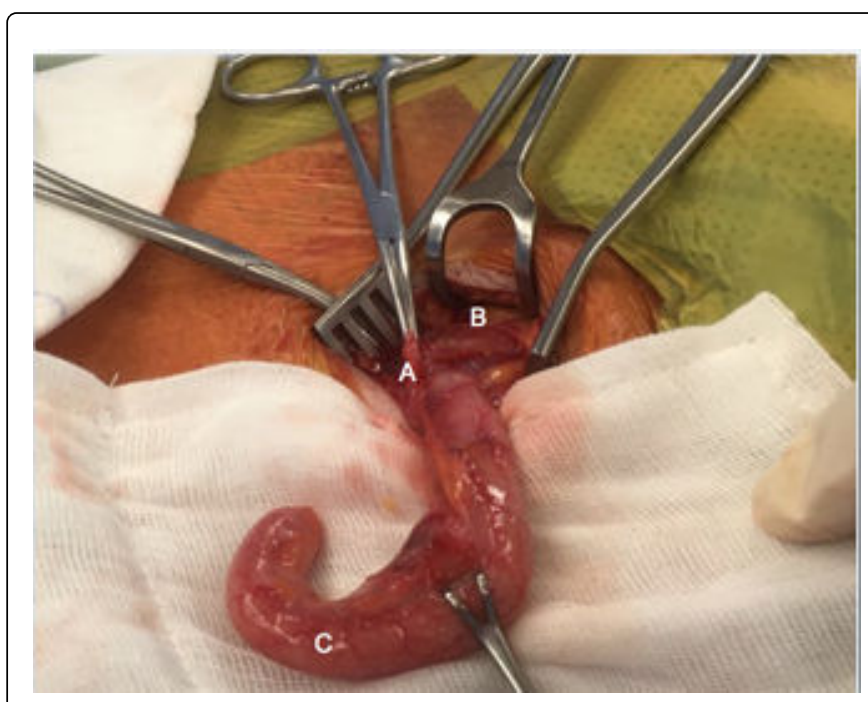

Figure 1: Indirect sac (A), Cord structures separated from the sac (B) and vermiform appendix (C).

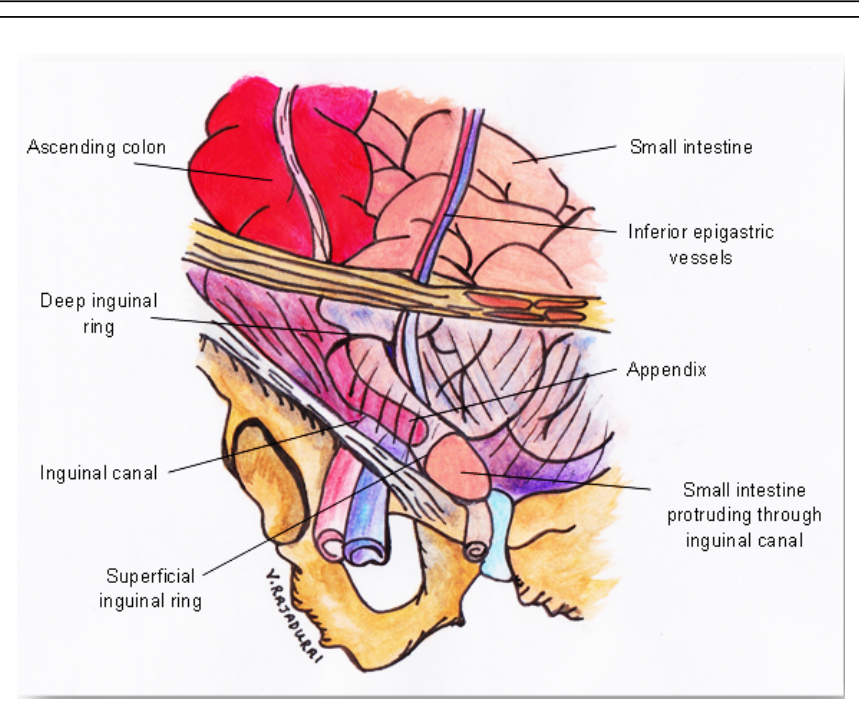

Figure 2: The schematic diagram depicts an Amyand's hernia with the appendix shown within an indirect inguinal hernia passing through the deep and superficial inguinal rings. 
Citation: Rajadurai VA (2016) Amyands Hernia: An Unexpected Finding during Inguinal Hernia Repair. J Gerontol Geriatr Res 5: 327. doi:

Page 2 of 2

\section{Discussion}

With Amyand's hernia being exceedingly rare and the incidence of an associated inflamed appendix being just slightly over 0.1 percent, diagnosis of an Amyand's hernia remains primarily an incidental finding during surgery. It usually occurs on the right side due to the normal anatomic position of the appendix, however there have been reported cases of Amyand's hernia on the left side, which has been suggested to be associated with intestinal malrotation, situs inversus or a mobile cecum $[4,5]$.

Some literature has suggested that in Amyand's hernia, the appendix becomes more vulnerable to trauma and is retained within the sac by adhesions. The inflamed appendix is then caused by compression of the appendix from abdominal muscle contractions or other increases in intra-abdominal pressure which significantly reduce or cut off its blood supply [6].

A proposed classification scale of Amyand's hernia (Type 1-4) has been described by Lasanoff and Basson to aid management $[7,8]$. Type 1 which describes a normal appendix within the sac should be managed by hernioplasty without appendectomy and Types 2-4 describe acute appendicitis within the sac which requires appendectomy followed by hernia repair without mesh [7]. Following this proposed classification, our patient had a Type 1 Amyand's hernia, however underwent an appendectomy. The decision to remove the non-inflamed appendix is one that is currently dictated primarily by the surgeon's preference.
In summary, Amyand's hernia is a rare form of inguinal hernia and presents as a diagnostic challenge given its low incidence and indistinct clinical presentation. Surgical intervention is usually diagnostic as well as therapeutic. Given that hernia repairs are one of the most common operations performed, it is important to have extensive knowledge of the anatomy and variants of hernias.

\section{References}

1. Amyand C (1736) Of an inguinal rupture, with a pin in the appendix caeci, incrusted with stone; and some observations on wounds in the guts. Philos Trans R Soc Lond 39: 329-336.

2. Ali S, Malik K, Al Qadhi H (2012) Amyand's Hernia: Study of Four Cases and Literature Review. SQU Medical Journal 12: 232-236.

3. Sharma H, Gupta A, Shekhawat N, Memon B, Memon M (2006) Amyand's hernia: a report of 18 consecutive patients over a 15 -year period. Hernia 11: 31-35.

4. Gupta S, Sharma R, Kaushik R (2005) Left-sided Amyand's hernia. Singapore Med J 46: 424-425.

5. Johari HG, Paydar S, Davani SZN, Eskandari S, Johari MG (2009) Leftsided Amyand hernia. Annals of Saudi Medicine 29: 321-322.

6. Solecki R, Matyja A, Milanowski W (2003) Amyand's hernia: a report of two cases. Hernia 7: 50-51.

7. Losanoff JE, Basson MD (2007) Amyand hernia: what lies beneath-a proposed classification scheme to determine management. Am Sur 73: 1288-1290.

8. Losanoff JE, Basson MD (2008) Amyand hernia: a classification to improve management. Hernia 12: 325-326. 